\title{
Relevante Daten als Schlüssel zum Erfolg
}

\author{
Bruno Soltermanna , Luzi Dubs ${ }^{b}$
}

${ }^{a}$ Facharzt für Chirurgie, Chefarzt SVV, Zürich; ${ }^{b}$ Facharzt für Orthopädische Chirurgie und Traumatologie am Bewegungsapparat, Winterthur

\author{
Die Qualität einer versicherungsmedizinischen, -technischen und medizinischen \\ Fallführung wird massgeblich durch die Dokumentation medizinischer Daten be- \\ einflusst. Enthält diese die klinisch wichtigen Daten, erfolgt die Fallführung kom- \\ petent und effizient.
}

Eine einwandfreie und umfassende medizinische Dokumentation ist für die medizinische wie auch für die versicherungsadministrative Fallführung wichtig. In medizinischer Hinsicht gilt es, den Patienten in Bezug auf die für seine krankheits- oder unfallbedingte Körperschädigung wichtigen Faktoren wie Anamnese, Klagen und Befunde zu erfassen und daraus dann eine Diagnose mit der entsprechenden Therapie abzuleiten. Insbesondere die aus der Körperschädigung resultierenden Fähigkeitsstörungen im Alltag, im Beruf und bei der Sportausübung sind für die weiteren Entscheidungen von vorrangiger Bedeutung. Für die Beurteilung der Arbeitsfähigkeit ist das noch vorhandene funktionelle Leistungsprofil mit den Anforderungen des Arbeitsplatzes zu vergleichen und daraus dann zum einen die Tätigkeitsbelastung und zum anderen der zeitliche Einsatz festzulegen [1].

Für den versicherungsadministrativen Teil sind zur Beurteilung der Leistungspflicht durch den Krankenoder Unfallversicherer insbesondere die Anamnese, die Befunde und die Diagnose wichtig, um bestmöglich Klarheit zu schaffen, ob ein Schadensmechanismus mit dem Ausprägungsgrad des funktionellen und morphologischen Schadensbildes nachvollziehbar übereinstimmt.

Um sich zudem aus versicherungsmedizinischer Sicht ein Bild des Versicherten machen zu können, sind in der Anamnese nicht nur der Krankheitsbeginn mit dessen Verlauf oder das traumatische Ereignis, sondern auch die familiäre und persönliche Anamnese mit der medizinischen Vorgeschichte und den Vorbefunden (Disposition) und die Angaben über eine berufliche oder sportliche Exposition von grosser Bedeutung. Diese Faktoren sind wesentliche Bestandteile für die Beurteilung der natürlichen Kausalität in Bezug auf die Anerkennung eines Leistungsfalles oder der Leistungsterminierung in der Unfallversicherung nach UVG. Speziell erforderlich sind diese Angaben seit Inkrafttreten der UVG-Revision vom 1.1.2017, die dem Unfallversicherer eine Beweislastumkehr beschert hat, indem dieser zwecks Entlastung von der Leistungspflicht nachweisen muss, dass es sich bei Vorliegen einer Listendiagnose "vorwiegend" um eine krankhafte oder degenerative Veränderung handelt (UVG Art. 6 Abs. 2). Die Ärzteschaft ist seither noch vermehrt verpflichtet, sich in ihrer Dokumentation der Krankengeschichte präzis und umfassend zu äussern.

\section{Wichtigkeit der Erstdokumentation}

\section{Anamnese}

Durch die Erfragung der familiären und insbesondere der persönlichen und sozialen Anamnese können medizinische Vorzustände wie aber auch Ressourcen oder Hindernisse für die Reintegration in Erfahrung gebracht werden.

\section{Schadensmechanismus}

Durch eine detaillierte Erfragung des Schadensmechanismus kann dem Untersucher bewusster werden, was genau vorgefallen ist und welche Kräfte auf welche Strukturen eingewirkt haben, was im Zusammenhang mit versicherungsmedizinischen Kausalitätsabklärungen erforderlich ist. Dabei kann sich der Leistungserbringer bereits fragen, ob die Schilderungen das Zustandsbild erklären können.

\section{Morphologisches Schadensbild}

Bei der Beurteilung des morphologischen Schadensbildes geht es darum, die Organschädigung in einer umfassenden klinischen Untersuchung mit Einbezug von Funktionstests und bedarfsgerecht in einer bildgebenden oder labormässigen Zusatzuntersuchung zu erfassen. Diese Beurteilung kann zusammen mit dem 
Schadensmechanismus klare Hinweise für die Kausalitätsbeurteilung ergeben.

\section{Funktionelles Schadensbild}

Das funktionelle Schadensbild kann zum einen über den Fähigkeitsverlauf nach einem Ereignis und allfällige Schwierigkeiten Auskunft geben, zum anderen aber auch im weiteren Verlauf direkt aufzeigen, was der Patient im Sinne der Fähigkeitsstörungen nach ICF aktuell kann oder nicht mehr respektive noch nicht wieder kann. Dies ist letztlich auch für die Beurteilung der Arbeitsunfähigkeit essentiell.

\section{Diagnose}

Handelt es sich um eine gesicherte Diagnose, so soll diese nach der ICD-Klassifikation (aktuell ICD 10) benannt werden.

Handelt es sich um eine unsichere Diagnose, so ist die Indikation für die weiteren diagnostischen Schritte immer mit der Vor- und Nachtestwahrscheinlichkeit der entsprechenden Untersuchung zu überdenken.

\section{Beurteilung der Arbeitsunfähigkeit}

Der Fähigkeitsverlauf soll aufzeigen, was der Patient kann oder nicht mehr respektive noch nicht wieder kann. Dieses Leistungsprofil soll in Bezug zum Anforderungsprofil des Arbeitsplatzes gestellt werden, um das Ausmass der Arbeitsunfähigkeit zu begründen.

\section{Kausalitätsbeurteilung durch den Versicherungsmediziner}

Die Leistungspflicht eines Unfallversicherers oder eines Haftpflichtversicherers setzt voraus, dass zwischen dem Unfall bzw. dem schädigenden Ereignis und dem eingetretenen Schaden eine natürliche Kausalität besteht. Ursachen im Sinne der natürlichen Kausalität sind alle Umstände, ohne deren Vorhandensein der eingetretene Erfolg nicht als eingetreten oder nicht als in der gleichen Weise bzw. nicht zur gleichen Zeit eingetreten gedacht werden kann. Es geht hierbei also darum, zu beurteilen und zu beantworten, ob die geklagten Beschwerden und die Befunde Unfallfolgen oder Krankheit sind. Dazu sind alle medizinischen Beweismittel pro und contra indizienhaft darzulegen, dies unter Berücksichtigung der gesamten medizinischen Faktenlage, bei der dem Erstbericht eine wesentliche Bedeutung zukommt, da der Erstbericht die «Aussagen der ersten Stunde» beinhalten muss, welche in der Regel unbefangener und zuverlässiger sind als spätere Darstellungen, die bewusst oder unbewusst von nachträglichen Überlegungen versicherungsrechtlicher oder anderer Art beeinflusst sein können.

\section{Dokumentationsqualität}

Im April 2018 wurde durch den Chefarzt des Schweizerischen Versicherungsverbandes eine Umfrage zur Dokumentationsqualität bei den auf der Website der Swiss Insurance Medicine (SIM) aufgelisteten 75 SIMzertifizierten Gutachterinnen und Gutachtern mit dem Facharzttitel für Orthopädische Chirurgie und Traumatologie des Bewegungsapparates durchgeführt [2].

Die Auswahl fiel auf zertifizierte Gutachterinnen und Gutachter, da diese sich bei Gutachten immer wieder auf Erstberichte abstützen müssen und darum am meisten Erfahrung mit der Beurteilung solcher Berichte haben.

Für die Umfrage wurde das Onlinetool www.online umfragen.com verwendet.

Bei der Befragung ging es lediglich um die Beurteilung orthopädischer Erstberichte, auch wenn der Patient bereits durch andere Mediziner beurteilt wurde.

Die Umfrage war vollkommen anonymisiert. Es bestand zu keinem Zeitpunkt die Möglichkeit, Rückschlüsse auf die teilnehmenden Orthopädinnen und Orthopäden zu ziehen.

Die Auswertung konnte direkt innerhalb des Onlinetools gemacht werden.

Von den 75 angeschriebenen SIM-zertifizierten Gutachterinnen und Gutachtern haben 35 (47\%) alle Fragen beantwortet.

Die Auswertungen zeigten, dass beinahe 60\% der orthopädischen Erstberichte von den medizinischen Gutachterinnen und Gutachtern mit Facharzttitel für Orthopädische Chirurgie und Traumatologie des Bewegungsapparates als mässig bis schlecht beurteilt wurden. Was zudem aufhorchen lässt, ist das sehr schlechte Abschneiden der Beurteilung des funktionellen Schadensbildes und damit vergesellschaftet der Beurteilung der Arbeitsunfähigkeit, denn hier werden enorme Versicherungsleistungen durch Taggelder unsachgerecht ausgelöst. Der Schadensmechanismus wird eher nicht erfragt, was zusammen mit den anderen mässig bis schlecht zu bezeichnenden Parametern dazu führt, dass die Kausalität schlecht beurteilbar ist. Unter den gleichzeitig geäusserten Bemerkungen wird mehrmals erwähnt, dass hier die Hausärzte eine wesentliche Rolle für die schlechte Berichtsqualität mitspielen und dass man sich auch überlegen könnte, für die Versorgung von Unfallpatienten eine besondere fachliche Qualifikation erlangen zu müssen, so wie es z.B. in Deutschland mit dem Durchgangsarzt ist. Dies wäre in der Schweiz wohl ein äusserst langwieriger standespolitischer und auch gesellschaftspolitischer Prozess. 
Die Ärzte sollten sich zudem mehr Zeit für Anamnese, klinische Untersuchung und Dokumentation nehmen und auch Angaben zum neurovaskulären Status machen.

\section{Checkliste minimale Dokumentation in Traumatologie}

Aufgrund dieser doch eher als insuffizient zu bezeichnenden Qualität der Erstdokumentation gibt es verschiedene Verbesserungsvorschläge, allen voran eine Checkliste für die minimale Dokumentation bei orthopädisch-traumatologischen Fällen. Die Daten können allesamt in die von den Versicherern einverlangten Arztberichte eingetragen werden [3], allenfalls wird auch eine Kopie der Krankengeschichte von den Versicherern akzeptiert, wenn hieraus die minimal geforderten Angaben ersichtlich sind; das würde den administrativen Aufwand für alle Beteiligten vermindern. Nebst der Einführung dieser Checkliste sollten versicherungsmedizinisch ausgerichtete Fortbildungen an Kongressen und Tagungen durchgeführt werden, an denen die Wichtigkeit der fundierten ärztlichen Dokumentation mit Schadensmechanismus, morphologischem und funktionellem Schadensbild, Überlegungen zur Indikation in diagnostischer und therapeutischer Hinsicht sowie die Beurteilung der Arbeitsunfähigkeit und die Überlegungen zur natürlichen Kausalität dargelegt werden.

Zudem sind auch die Hausärzte vermehrt für die Untersuchung, Beurteilung und Dokumentation des muskuloskelettalen Schadensmechanismus und des Schadensbildes weiter- und fortzubilden. Die Schweizerische Gesellschaft für Traumatologie und Versicherungsmedizin (SGTV) hat schon Vorarbeit geleistet [4].

Checkliste minimale Dokumentation in Traumatologie und Orthopädie für den Erstbericht.

\section{Anamnese}

Patient, Soziales, Familie

Die Literatur findet sich unter www.saez.ch

$\rightarrow$ Aktuelle Ausgabe oder $\rightarrow$ Archiv $\rightarrow 2019 \rightarrow 21$

Korrespondenz:

Dr. med. Bruno Soltermann, MAS Versicherungsmedizin Chefarzt SVV

Conrad-Ferdinand-Meyer-

Strasse 14

Postfach

CH-8022 Zürich

Tel. 0442082865

bruno.soltermann[at]svv.ch
Checkliste minimale Dokumentation für den Verlaufsbericht, bezogen auf den geschädigten und den zu dokumentierenden Organbereich mit Angaben über den Fähigkeitsverlauf in Alltag, Beruf und Sport.

\begin{tabular}{ll}
\hline Körperregion & Minimaldokumentation \\
\hline Schulter bis Hand & Komplexfunktionen \\
& Beweglichkeit \\
& Kraft \\
\hline Hüfte & Gangbild \\
& Beweglichkeit \\
& Kraft \\
\hline Knie & Gang, Kauern, Einbeinstand \\
& Beweglichkeit \\
& Erguss \\
& Stabilität \\
& Muskelumfang am Oberschenkel \\
& beidseits \\
\hline Fuss & Komplexe Gangproben \\
& Beweglichkeit \\
& Stabilität \\
\hline Wirbelsäule & Komplexfunktionen \\
& Beweglichkeit \\
\hline
\end{tabular}

Es sind von methodischer Seite Anstrengungen zu unternehmen, verschiedene klinische und medizintechnische Tests (Röntgen, Labor, Funktionstests) auf ihre Testeigenschaften wie Sensitivität, Spezifität, Likelihood Ratio usw. zu überprüfen und einzuordnen, um sie gemäss ihrem Informationsgewinn in der Diagnostik einsetzen zu können. Von speziellem Wert sind die sogenannten SnNOut- oder SpPIn-Tests nach Sackett et al. [5]. Bei negativem Resultat und hoher Sensitivität kann eine Zielerkrankung ausgeschlossen (SnNOut = Sensitivität hoch, Test negativ, Krankheit Out) bzw. bei positivem Resultat und hoher Spezifität (SpPIn = Spezifität hoch, Test positiv, Krankheit In) eine solche nachgewiesen werden. Damit lässt sich eine Verminderung des diagnostischen und somit auch administrativen Aufwandes erreichen.

\section{Fazit}

Durch eine gute medizinische Dokumentation kann die medizinische, versicherungsmedizinische und -technische Fallführung kompetent und effizient erfolgen. Es sollen nicht möglichst viele, sondern die klinisch wichtigen Daten dokumentiert werden.

Hierdurch werden Missverständnisse und Verständigungsschwierigkeiten in der Korrespondenz mit Versicherungsfachleuten und Versicherungsmedizinern abgebaut, und der administrative Aufwand in der täglichen Praxis wird verringert. Die Versicherer können ihre Leistungspflicht besser und schneller abklären. Zudem können vollständige Basisdokumentationen für die Forschung verwendet werden. 